\title{
An Fast Refined Algorithm for Image Mosaic
}

\author{
FENG Yu-ping \\ College of Automation and Electronic Engineering \\ Qingdao University of Science and Technology \\ Qingdao, China \\ gjsfengyuping@163.com
}

\author{
ZHAO Wen-cang \\ College of Automation and Electronic Engineering \\ Qingdao University of Science and Technology \\ Qingdao, China \\ wencangzhao@gmail.com
}

\begin{abstract}
In order to improve accuracy and speed of image mosaic, an optimized algorithm of image mosaic based on corners is presented. Frequency phase correlation is used to estimate the overlapping area, in which improved Harris operator is used to extract corners. Then, rough and RANSAC accurate matching will be completed. Finally, image variance combined linear weight function is used to implemented image fusion and mosaic, and it presents quantitative analysis method about registration accuracy. Experimental results show that this algorithm exceeds existing ones at matching speed about $40 \%$, and has a desired visual effect. The MAD and RMSE of corresponding points is within 1 pixel precision.
\end{abstract} fusion

Keywords- image mosaic; corner detection; RANSAC; image

\section{INTRODUCTION}

Image mosaic is used to improve the visual filed of common camera which can not shoot an wide panoramic image once. Image mosaic is the art of registering and stitching some overlapped pictures into a bigger one, which is a high-resolution image with large visual angle, the whole information of multiple images and good visual effect. Image mosaic is an important research topic, and has been widely used in photogrammetry, virtual reality, video compression, video index, and so on.

Image mosaic algorithm is mainly divided into three categories based on image registration:the method based on image pixels;the method based on image characteristics;the method based on frequency domain. Image mosaic includes three steps:image pre-processing, image registration, image fusion ${ }^{[1-5]}$. The image mosaic method based on the characteristics is a class of the most widely used, and has a best effect. At present, various image stitching algorithms mainly have two problems. The speed relatively can not meet the real time requirements; the stitching accuracy can not be quantifiably measured and registration precision is relatively low. So, this paper presents an fast refined image mosaic algorithm. Experimental results show that this algorithm has an high matching speed and precision,which has a desired visual effect.

\section{CORNER DETECTION AND MATCHING}

\section{A. Harris extraction}

During the most feature point extraction algorithms, Harris corners detector has an excellent performance and is used in this paper ${ }^{[6,7]}$. Denoting the image intensities by I, the change produced by a shift( $(\mathrm{u}, \mathrm{v})$ is given by:

$$
E(u, v)=\sum_{x, y} w(x, y)[I(x+u, y+v)-I(x, y)]^{2}
$$

where $w(x, y)$ specifies the image window, we use a smooth circular window for noise reduction, for example a Gaussian:

$$
w(x, y)=\exp \left[-\left(x^{2}+y^{2}\right) / 2 \sigma^{2}\right]
$$

The change $\mathrm{E}$, for the small shift can be concisely written as

$$
\begin{gathered}
E(u, v) \cong\left[\begin{array}{ll}
u & v
\end{array}\right] M\left[\begin{array}{l}
u \\
v
\end{array}\right] \\
M=\sum_{x, y} w(x, y)\left[\begin{array}{cc}
I_{x}{ }^{2} & I_{x} I_{y} \\
I_{x} I_{y} & I_{y}{ }^{2}
\end{array}\right]
\end{gathered}
$$

Where $I_{x}$ an $I_{y}$ represent the image intensity gradients in the horizontal and vertical directions. Note that $\mathrm{E}$ is closely related to the local autocorrelation function. Let $\lambda_{1}$ and $\lambda_{2}$ be the eigenvalues of $\mathrm{M}$. If $\lambda_{1}$ and $\lambda_{2}$ are both high, then shifts in any direction will increase $E$ : this indicates a corner. So consider the following inspired formulation for the corner response:

$$
R=\operatorname{det} M-k(\text { trace } M)^{2}
$$

where $\operatorname{det} M=\lambda_{1} \lambda_{2}, \quad$ trace $M=\lambda_{1}+\lambda_{2}, \mathrm{k}$ is an empirical parameter $(k=0.04 \sim 0.06)$. A corner region pixel is selected when the $\mathrm{R}$ is a local maximum and greater than the given threshold $\mathrm{T}$.

\section{B. Improved Harris}

As before, there are three cases about Harris for image mosaic to be considered:1. Parameter $\mathrm{k}$ is empirical, and its selection has an randomness. 2.Threshold $\mathrm{T}$ needs to be given by myself. If $\mathrm{T}$ is smaller, excessive points will be extracted and increase the image matching calculation. Characteristic of partial points is insufficient, the matching will be affected. If $\mathrm{T}$ is larger, little points will be extracted and decrease the image matching success rate. Attributes, color, texture and structure characteristics vary greatly for different kinds of images, so that appropriate threshold also vary a lot. So this paper presents some improved measures.

1.Improved Harris response function:considering $R$ is a difference between $\operatorname{det} M$ and trace $M$, so the function can be changed using mathematical principle,as follow:

$$
R=\operatorname{det} M /(\text { trace } M+\varepsilon)
$$


$\mathrm{R}$ is joined a minimum in the denominator in order to prevent zero divisor overflowing . So the ratio $\mathrm{R}$ has a non randomness, also has practicality, reliability.

2.Auto-confirming the threshold $\mathrm{T}$ according the first image:setting a relatively small initial threshold $T_{0}$ for the first image within image sequence, extracting local maximum within the corners greater than $T_{0}$, sorting the results, selecting some corners as candidate corners and recording the minimal threshold as the $\mathrm{T}$ for the other images. So the number of feature points is reasonable, and the threshold can also be automatically selected, the speed has greatly increased as extracting once.

3.Sub-pixel accurate positioning corners: fitting approximation corner response function $\mathrm{R}$ by using polynomial $a x^{2}+b y^{2}+c x y+d x+e y+f=R(x, y)$, complete subpixel accurate positioning. The unknown polynomial parameters $(a, b, c, d, e, f)$ are solved by composing the equation groups using the 8 neighborhood pixel response of candidate corners. The maxima of polynomia is subpixel corner.

Frequency phase correlation is used to estimate the overlapping area, in which improved Harris operator is used to extract corners, this can improve the speed avoiding the invalid computation in the full area image.

\section{Features matching}

After corner detection, we should estimate registration parameters between two images by image feature matching. The Harris corner matching usually is accomplished using the gray information of feature neighborhood. In this paper, the initial feature point pairs are obtained with bidirectional greatest correlative coefficient, and a few false feature point pairs are rejected by RANSAC ${ }^{[8]}$.

The rough matching process is as follows: The approximate position of corresponding corner in second image is calculated according to the translation and the corner position in first image. Within the $40 \times 40$ neighborhood of approximate position, we search possible matching points, and the reverse is equally true. The intersection is taken as the coarse matching results. So the speed can increase greatly by decreasing the searching region, and the matching success rate also is enhanced. As before, the matching method is relatively robust with image noise and repetitive texture. Correlative matching coefficient function is as follows:

$$
N C C(i, j)=\frac{\sum_{x=1}^{2 N+12 N+1}\left[I_{y=1}(x, y)-\overline{I_{1}}\right]\left[I_{2}(x, y)-\overline{I_{2}}\right]}{\sqrt{\sum_{x=1}^{N+12 N+1} \sum_{y=1}\left[I_{1}(x, y)-\overline{I_{1}}\right]^{2}} \sqrt{\sum_{x=1}^{2 N+12 N+1}} \sum_{y=1}^{2}\left[I_{2}(x, y)-\overline{I_{2}}\right]^{2}}
$$

In the equation (7), $\bar{I}_{1}$ and $\overline{I_{2}}$ are the gray average of correlative window, we take the corners which have the greatest coefficient and greater than threshold as the results.

Pseudo features after rough matching can influence image mosaic, we do image RANSAC refine matching for getting rid of pseudo features. Precise matching detailed process can be seen in reference [8].

\section{Analysis of registration accuracy}

We usually do not know the true registration parameters, so the geometric error of image registration can not be directly quantitative analysed. In order to get the registration accuracy, MAD (Mean Absolute Difference) and RMSE

( Root Mean Square Error) of transformed matching points are presented as evaluating indexes, the equation is as follows:

$$
\begin{gathered}
M A D=\frac{\sum_{i=1}^{n}\left\|X_{1 i}-M X_{2 i}\right\|}{n} \\
R M S E=\sqrt{\frac{\sum_{i=1}^{n}\left\|X_{1 i}-M X_{2 i}\right\|^{2}}{n}}
\end{gathered}
$$

\section{IMAGE FUSION AND MOSAIC}

The input image needs to be transformed with a same coordinate system of the reference image after image registration. If we just take one picture information in overlap region, splicing apertures may be brought. When the two image have an obvious luminance difference, the image mosaic will be untrue. So in this paper, linear weight function combined image statistical characteristics is used to implement image fusion and mosaic.

We adjust the image luminance using mean and variance which are the statistical parameters. The statistical characteristics of the first image are assigned to the second image. The adjustment process is as follows:

$$
\left\{\begin{array}{l}
\widetilde{I}_{1}=I_{1} \\
\widetilde{I}_{2}=I_{2}+\left(\overline{M_{2}}-\overline{M_{2}}\right)
\end{array}\right.
$$

Where $\widehat{M_{2}}=M_{2}-\overline{M_{2}}, \widetilde{M_{2}}=\widehat{M_{2}}\left(\sigma_{1} / \sigma_{2}\right)+\overline{M_{1}}$. So $\widetilde{I_{2}}$ and $\widetilde{I}_{1}$ have the same brightness. Linear weighted fusion is used to implement image mosaic.

\section{EXPERIMENT RESULTS AND ANALYSIS}

The experimental machine is P4 CPU 2.4G, memory $1 \mathrm{~GB}$, the software programming environment is MATLAB 7.0. The corners detection and matching results of two aerial images are shown in Fig.1. Fig.2 is the mixture image of two.The MAD and RMSE of corresponding points is 0.3346 and 0.3676. Registration geometric error is within 1pixel. Accuracy is relatively high.

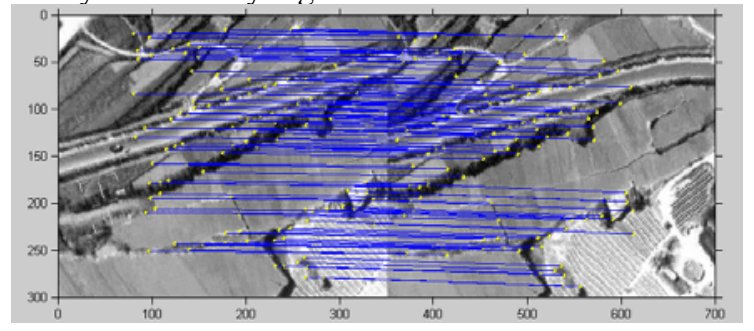

Figure 1. Feature detection and matching results of two registering images 


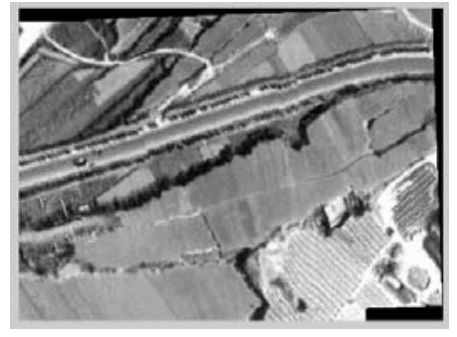

Figure 2. Mixture result of two registered image

In Fig.3, we take a image sequence to verify the algorithm. The corner detection results in overlap region are shown with the number of 41 and 40 in Fig.4. We get mosaic image using the method of this paper. Comparing the time of single corner detection and matching, the average time in Fig. 3 is 1.052 seconds, the average time of unimproved algorithm is 2.048 seconds. The average time of improved algorithm has increased $50 \%$ based on the experiment with all kinds of pictures. The fusion image is shown in Fig.5. The color panorama with video is given in Fig.6. We can see that the mosaic image have the color consistency, no splicing apertures, no brightness jumpment.

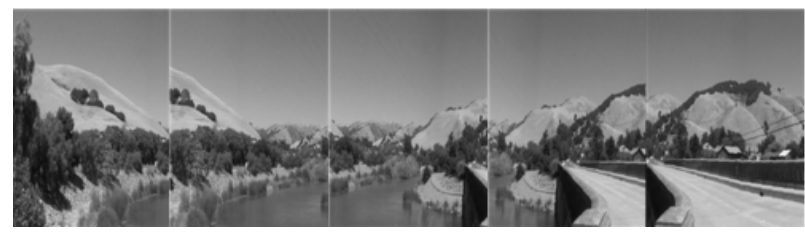

Figure 3. Registering image sequence

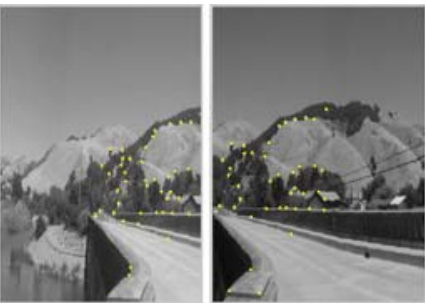

Figure 4. Corner detection

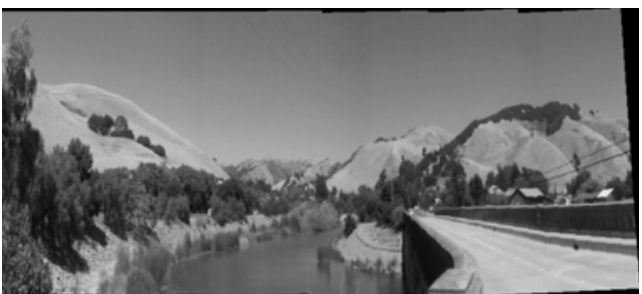

Figure 5. Statistical weighted fusion and mosaic

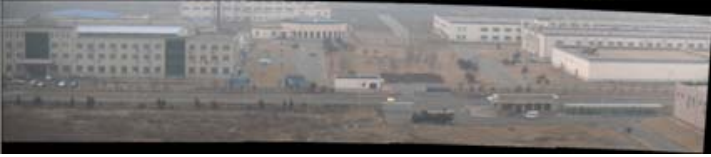

Figure 6. Color panorama

\section{CONCLUSIONS}

An fast refined image mosaic algorithm based on corners is presented. The speed and accuracy both are enhanced because the improved corner extraction and matching algorithm. The linear weight function based on statistical characteristics makes the fusion image nature.The whole image mosaic is automatic and fast. Experiment results show that this algorithm has an high matching speed and precision, which has a desired visual effect and robustness.

\section{ACKNOWLEDGMENT}

This work is supported by the Chinese Nature Science Foundation ( 61171131$)$ and SAQSIQ Project (2010IK232).

\section{REFERENCES}

[1] B ZITOVA, J FLUSSER, "Image registration methods : a survey,’'Image and Vision Computing,2003, vol.21(11),pp.977-1000.

[2] R Szeliski, “Image Alignment and Stitching: A Tutorial,’'Richard Szeliski Preliminary draft, January 26, 2005, Technical Report, MSR-TR-2004-92.

[3] L H CAI, L H LIAO, D H GUO, "Study on image stitching methods and its key technologies,' Computer Technology and Development, 2008, vol.18(3),pp.1-4.

[4] Y Y GAO,J F YANG,X L MA, “Interference image registration based on Fourier-Mellin algorithm,”.Optics and Precision Engineering,2007,vol.15(9) ,pp.1415-1420.

[5] J HE , Y S LI, H LU H, "Research of UAV Image Mosaic Based on SIFT,”Opto-Electronic Engineering,2011,vol.38(2) ,pp.122-126.

[6] J ZHU,M W REN,YANG Z J, "Fast Matching Algorithm Based on Corner Detection ," Journal of Nanjing University of Science and Technology, 2011, vol.35(6),pp.755-758.

[7] G Y Tian, D Gledhill, D Taylor, “ Comprehensive interest points based imaging mosaic,” Pattern Recognition Letters,2003,vol.24,pp.1171-1179.

[8] M Fischler, R BSolles, "Random sample consensus:A paradigm for model fitting with applications to image analysis and automated cartography,"Communications of the ACM , 1981 , vol.24 (6) ,pp.381-395. 